\title{
Pentacam Scheimpflug Quantitative Imaging of the Crystalline Lens and Intraocular Lens
}

\author{
Patricia Rosales, PhD; Susana Marcos, PhD
}

\begin{abstract}
PURPOSE: To implement geometrical and optical distortion correction methods for anterior segment Scheimpflug images obtained with a commercially available system (Pentacam, Oculus Optikgeräte $\mathrm{GmbH}$ ).

METHODS: Ray tracing algorithms were implemented to obtain corrected ocular surface geometry from the original images captured by the Pentacam's CCD camera. As details of the optical layout were not fully provided by the manufacturer, an iterative procedure (based on imaging of calibrated spheres) was developed to estimate the camera lens specifications. The correction procedure was tested on Scheimpflug images of a physical water cell model eye (with polymethylmethacrylate cornea and a commercial IOL of known dimensions) and of a normal human eye previously measured with a corrected optical and geometrical distortion Scheimpflug camera (Topcon SL-45 [Topcon Medical Systems Inc] from the Vrije University, Amsterdam, Holland).
\end{abstract}

RESULTS: Uncorrected Scheimpflug images show flatter surfaces and thinner lenses than in reality. The application of geometrical and optical distortion correction algorithms improves the accuracy of the estimated anterior lens radii of curvature by $30 \%$ to $40 \%$ and of the estimated posterior lens by $50 \%$ to $100 \%$. The average error in the retrieved radii was 0.37 and $0.46 \mathrm{~mm}$ for the anterior and posterior lens radii of curvature, respectively, and $0.048 \mathrm{~mm}$ for lens thickness.

CONCLUSIONS: The Pentacam Scheimpflug system can be used to obtain quantitative information on the geometry of the crystalline lens, provided that geometrical and optical distortion correction algorithms are applied, within the accuracy of state-of-the art phakometry and biometry. The techniques could improve with exact knowledge of the technical specifications of the instrument, improved edge detection algorithms, consideration of aspheric and non-rotationally symmetrical surfaces, and introduction of a crystalline gradient index. [J Refract Surg. 2009;25:421-428.]

DOI:10.9999/1081597X-20090422-04

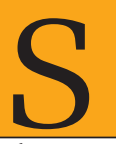

cheimpflug imaging is a powerful tool for imaging the anterior segment, but special care must be taken to correct the images from geometrical distortion (caused by tilt of the object plane with respect to the optical axis of the instrument) and from optical distortion (caused by refraction from the different ocular surfaces). ${ }^{1}$

The Scheimpflug principle has been applied to imaging the eye's anterior segment since the 1970s. ${ }^{2}$ Commercial instruments were available for some time in the $1980 \mathrm{~s}$ and $1990 \mathrm{~s}^{3-5}$ and the need to apply distortion correction algorithms had been reported. However, this type of imaging had not been widely used in clinical practice until 2005 when new instruments, such as the Pentacam (Oculus Optikgeräte GmbH, Wetzlar, Germany) and GALILEI dual Scheimpflug analyzer (Ziemer Ophthalmology, Port, Switzerland), were introduced. Despite the large depth of focus of Scheimpflug imaging-based systems capable of capturing cross-sections of the human eye from the anterior cornea to the posterior crystalline lens (particularly under full dilation), current commercial instruments are mostly used as corneal topographers and pachymeters, discarding quantitative information that could be extracted on the crystalline lens geometry.

From the Instituto de Óptica "Daza de Valdés," Consejo Superior de Investigaciones Científicas, Madrid, Spain.

This study was supported by Ministerio de Educación y Ciencia Grant FIS2005-04382 (Marcos); EURYI Award (Marcos); and FPI Predoctoral Fellowship BFM2002-02638 (Rosales).

The authors have no proprietary interest in the materials presented herein.

The authors thank Michiel Dubbelman, PhD, and Rob van der Heijde, PhD, for helpful discussions on the correction algorithms and measurements with their Scheimpflug instrument; David Atchison, PhD, Robert Iskander, PhD, Sanjeev Kasthurirangan, PhD, Alfonso Pérez-Escudero, MSc, and Carlos Dorronsoro, $\mathrm{MSc}$, for implementing software to retrieve raw images from the Pentacam system; and Alberto de Castro, MSc, for implementing a geometrical distortion correcting algorithm.

Correspondence: Susana Marcos, PhD, Instituto de Óptica, CSIC, Serrano 121, 28006 Madrid, Spain. Tel: 34 915616800; Fax: 34 915645557; E-mail: susana@io.cfmac.csic.es

Received: February 28, 2008; Accepted: September 12, 2008

Posted online: October 15, 2008 
The first Scheimpflug imaging system used for investigation of the crystalline lens in vivo was developed by Brown. ${ }^{2}$ Although Brown introduced corrections for geometrical distortion, the optical distortion did not seem to be fully corrected. Differences across studies in the reported change of anterior and posterior lens radius can be attributed to different amounts of distortion correction. For example, Brown ${ }^{2}$ reported a decrease in the anterior lens radius of $100 \mu \mathrm{m} / \mathrm{year}$, whereas Dubbelman and van der Heijde ${ }^{6}$ report a decrease of $57 \mu \mathrm{m} /$ year.

Different correction methods have been applied to Scheimpflug imaging optical distortion correction. Cook and Koretz ${ }^{7}$ proposed a method based on a Hough transform. This method was validated by the same group through a comparison of anterior and posterior crystalline lens measurements with Scheimpflug imaging and magnetic resonance imaging. ${ }^{8}$ Both methods provided similar trends in the change of anterior and posterior radius of curvature measured on a different set of patients with each instrument.

Dubbelman et $\mathrm{al}^{6,9,10}$ developed correcting algorithms and validations on refurbished prototypes of the Topcon SL-45 (Topcon Medical Systems Inc, Paramus, NJ) and NIDEK EAS-1000 systems (NIDEK Co Ltd, Gamagori, Japan). These systems were commercially available in the past, although both are now discontinued. Dubbelman and colleagues performed hardware changes on these systems (including replacement of the original camera by a high-resolution scientificgrade CCD camera), implementation of new software and image capture protocols, and, in particular, ray tracing algorithms for geometrical and optical distortion correction. In a previous study, ${ }^{11}$ we compared anterior and posterior lens radii of curvature in the unaccommodated state and as a function of accommodation in a group of young eyes, measured using both the corrected Topcon SL-45 system and a Purkinje imaging system and found similar results. In both cases, images were taken along one meridian, which can be manually changed in orientation. Typically, data are obtained only on the horizontal and vertical meridians.

A popular Scheimpflug imaging system commercially available today is the Pentacam. The Pentacam images the anterior segment of the eye by a rotating Scheimpflug camera system. This rotating process allows rapid capture of images in different meridians, and therefore three-dimensional elevations. The Pentacam provides optical distortion-corrected data of the posterior cornea, although it does not perform any distortion correction on the crystalline lens surfaces. Although this system is primarily used as an anterior and posterior corneal topographer and pachymeter, further potential of the instrument relies on the capability of providing quantitative information on crystalline lens position and structure. We previously reported and validated measurements of intraocular lens tilt and decentration with the Pentacam Scheimpflug system, in comparison with Purkinje imaging on physical model eyes and pseudophakic eyes. ${ }^{12}$ We now report a method to obtain corrected anterior and posterior lens radii of curvature as well as lens thickness from distorted Pentacam Scheimpflug raw images.

\section{MATERIALS AND METHODS}

\section{SCheimpflug Principle}

We used a Pentacam anterior segment imaging system based on the Scheimpflug principle. The Scheimpflug camera is a modification of a slit-lamp camera, with a modified geometry to improve depth of focus. In a slit-lamp camera, the lens and image (film or sensor) planes of a camera are parallel to each other, and therefore the plane of focus is parallel to the lens and image planes. If a planar subject is also parallel to the image plane, it can coincide with the plane of focus, and the entire subject can be rendered sharply. If the subject plane is not parallel to the image plane, it will be in focus only along a line where it intersects the plane of focus. In a Scheimpflug camera, the slit beam, camera lens, and CCD sensor intersect in a line where a cross-section of the eye appears in focus.

\section{Optical Layout}

Figure 1 shows the optical layout of the Pentacam system as reconstructed from the specifications provided by the manufacturer upon request. Unlike the Topcon SL-45 and NIDEK EAS-1000, for which the critical parameters of the optical layout are available, the information from the Pentacam system is limited.

According to the manufacturer (personal communication, March 27, 2007), the lens and object form an angle $\alpha$ of $45^{\circ}$, and the image and lens plane form an angle $\beta<45^{\circ}$ (the actual amount was not provided). The application of the correction distortion algorithms requires knowledge of the camera's lens nodal points (or, assuming a thin lens, the object and image distances $a$ and $b$ ) and angle $\beta$. We followed an iterative method to retrieve $a, b$, and $\beta$. Calibrated spheres of known radii of curvature $(9.65,8$, and $6 \mathrm{~mm})$ were placed at different positions along the object plane and imaged with the Pentacam at a single meridian. Ray tracing was recursively performed with varying values of $a, b$, and $\beta$. A minimization procedure (mean least squares) was applied to obtain values that minimized the difference between the estimated and nominal radii of 


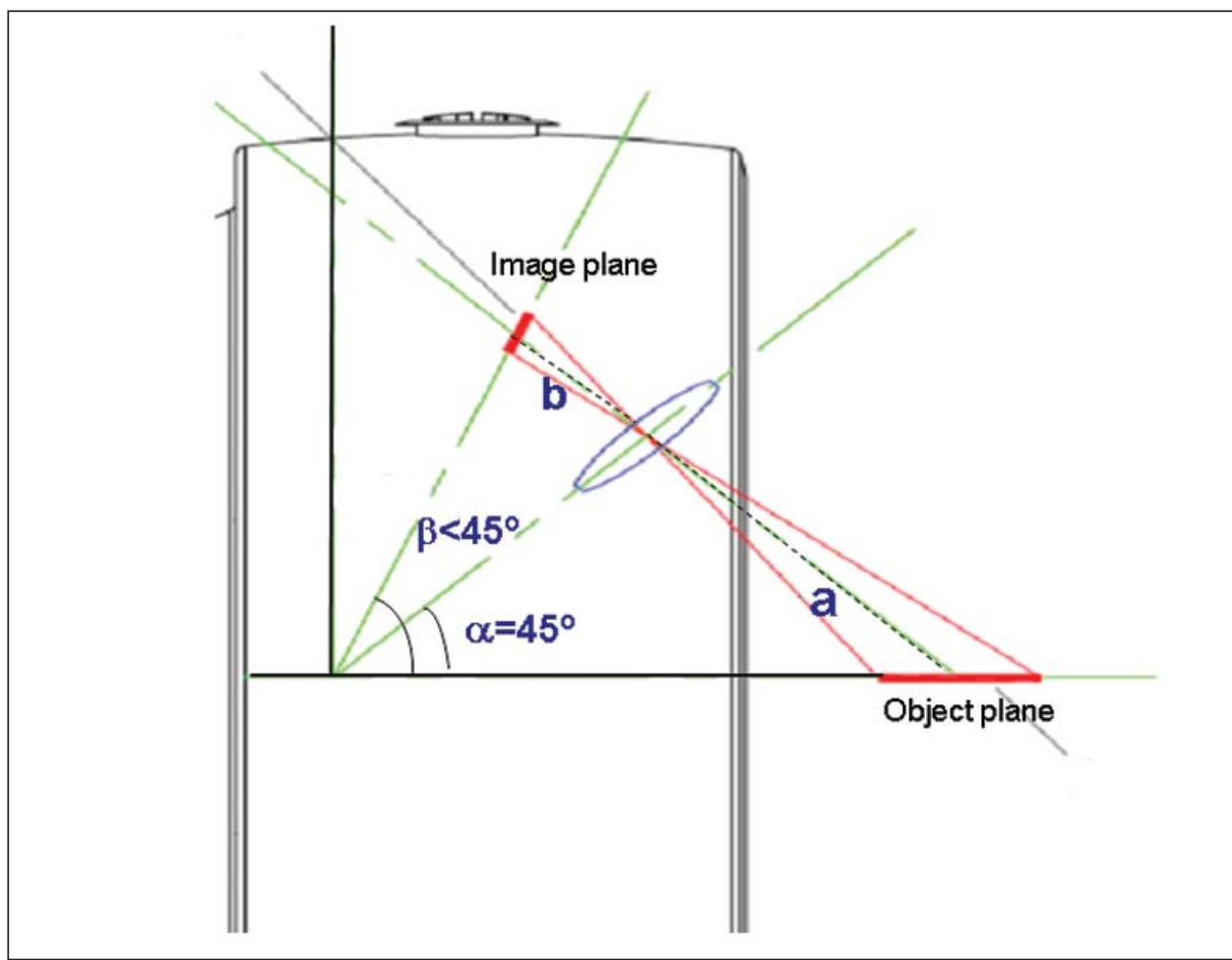

Figure 1. Schematic diagram of the optical layout of the Pentacam system, reconstructed from the specifications provided by the manufacturer. $a=$ object distance, $\mathrm{b}=$ image distance, $\alpha=$ angle between the lens and object plane, $\beta=$ angle between the lens and image plane

curvature of the calibrated spheres. As proof that the estimated nodal point was correct, a projection of two consecutive points from a test card (millimetric sheet) was obtained to check if the distance between two consecutive points projected was $1 \mathrm{~mm}$. The minimization routine was performed with randomized initial conditions for values of $a$ and $b$ between 63 and $250 \mathrm{~mm}$ (based on physical dimensions of the instrument) and a constraint of $\beta<45^{\circ}$ for the angle.

\section{RAW IMAGES}

The Pentacam stores raw images of the anterior segment of the eye in *.src files. However, the extraction of raw images as captured by the CCD camera from those files is proprietary and not provided by the manufacturer. Programs written in MATLAB (The MathWorks Inc, Natick, Mass) to retrieve the original raw images from the files were provided by David Atchison, Robert Iskander, and Sanjeev Kasthurirangan from the School of Optometry, Queensland University of Technology, Brisbane, Australia, and further adapted and refined by Alfonso Pérez-Escudero and Carlos Dorronsoro in our laboratory. Headers were discarded and the images exported to conventional file types for further processing. Typically, images were obtained at a single meridian using the single averaged image mode (averaging 15 images). Ocular edges, in most cases, were detected using a Canny filter. In some images with artificial lens (corneal or IOL) surfaces where the edges were not properly detected using the Canny filter, manual detection was used instead. The edges were fitted to circles using standard least-mean square procedures, with programs written in MATLAB.

\section{Distortion CORRECTION ALgORIthMS}

The reconstruction algorithms are based on those developed by Dubbelman et al $\mathrm{l}^{6,9,10}$ for the Topcon and NIDEK systems and adapted to the particular configuration of the Pentacam system.

The geometrical distortion was corrected by projecting the images captured on the CCD camera chip back to the object plane passing through the camera's lens optics, allowing retrieval of the real coordinates. The anterior corneal surface only suffers from the geometrical distortion. After correction, the edges of the anterior cornea cross-section are fitted to a circle, which is used to reconstruct the anterior corneal surface as a sphere.

The optical distortion was corrected by means of ray tracing. Figure 2 illustrates the ray tracing procedure. Assuming that the surface is rotationally symmetric, the posterior surface is traced through the camera lens optics nodal point and refracted by the anterior cornea, then projected on the object plane. The projected points are fitted to a spherical surface. Identical procedures are followed to reconstruct the anterior and posterior surface of the lens. Refractive indices of 1.49, 1.33, and 1.458 were used for the polymethylmethacrylate (PMMA) cornea, saline solution, and silicone IOL, respectively. Refractive indices of 1.376, 1.336, and 1.42 were used for the effective index of the hu- 


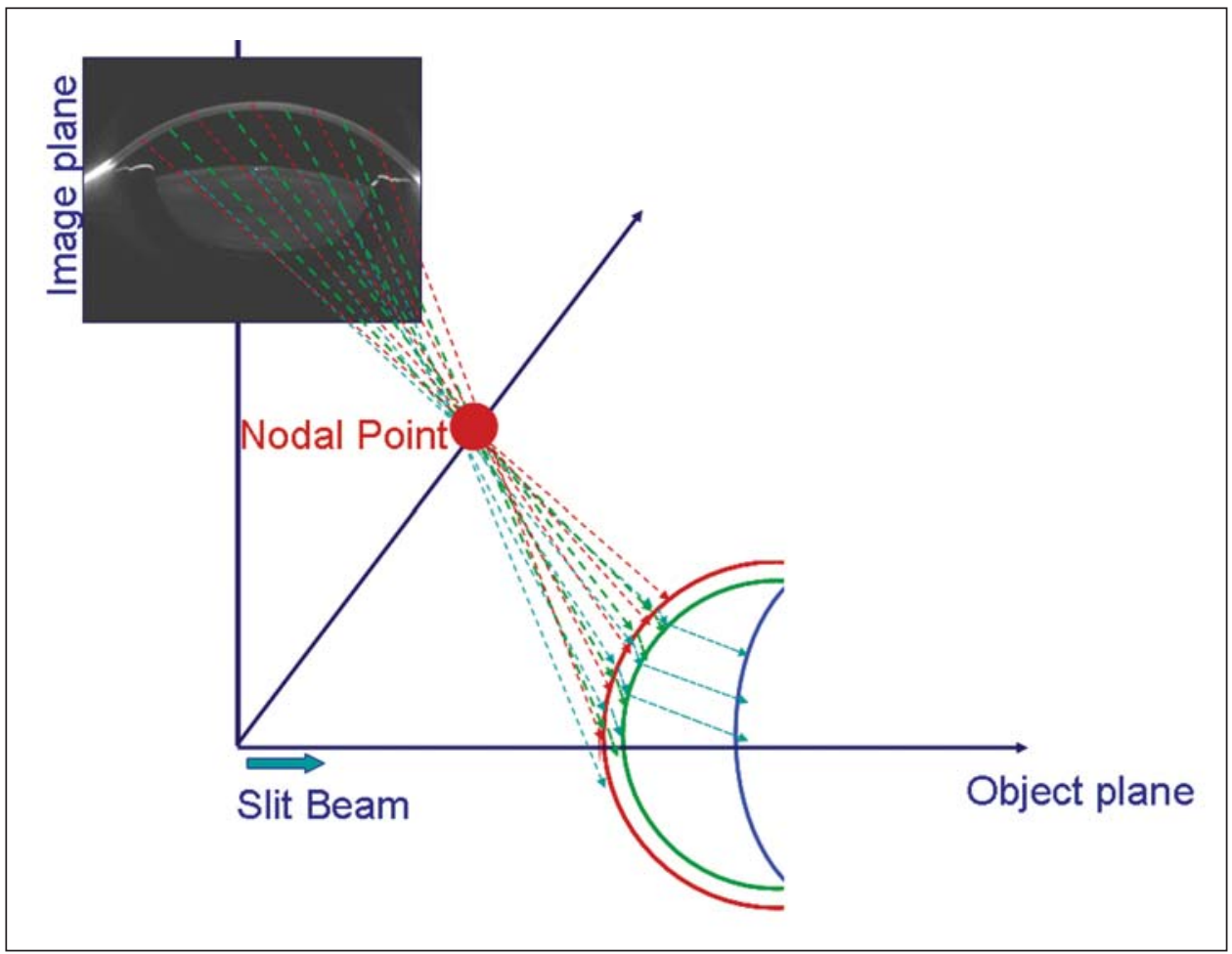

Figure 2. Schematic illustration of the sequential ray tracing through the nodal point of the camera lens performed to correct the optical distortion caused by the ocular components (posterior cornea by the anterior cornea, anterior lens by the anterior and posterior cornea, and posterior lens by anterior and posterior cornea and anterior lens).

TABLE 1

Nominal and Estimated Radii of Curvature and Interocular Distances From Uncorrected and Corrected Scheimpflug Images for a Physical Model Eye

\begin{tabular}{lccc}
\hline & Nominal Values $(\mathbf{m m})$ & Before Correction $(\mathbf{m m})$ & After Correction $(\mathbf{m m})$ \\
\hline Anterior corneal radius & 7.80 & 8.98 & 7.59 \\
Corneal thickness & 0.55 & 0.31 & 0.53 \\
Posterior corneal radius & 6.48 & 8.48 & 6.43 \\
Anterior chamber depth & 3.00 & 2.15 & 2.52 \\
Anterior lens radius & 12.25 & 15.85 & 11.68 \\
Lens thickness & 1.164 & 0.99 & 1.24 \\
Posterior lens radius & 12.25 & 24.26 & 11.59
\end{tabular}

man cornea, aqueous humor, and effective index of the human lens, respectively.

\section{TEST EYES}

The distortion correction algorithms were tested on two eyes-a physical model eye with known dimensions and a human phakic young eye.

The physical model eye consisted of a water cell model with a spherical PMMA contact lens simulating the cornea and an IOL simulating the lens. ${ }^{12}$ The "cornea" was built by a contact lens manufacturer (AR3 Vision, Madrid, Spain) with parameters similar to those of the Gullstrand eye model. We used a spherical IOL with known geometry and refractive index (CeeOn Edge
911, 19.00 diopters; Pharmacia Corp, Peapack, NJ). ${ }^{13}$ The first column of Table 1 shows the nominal parameters of the physical model eye.

The right eye of one of the authors (P.R., age 33, nearly emmetropic) was also used as a test. This subject had been previously measured using Purkinje imaging and a corrected Topcon SL-45. The first column of Table 2 shows the values obtained from this eye using the corrected Scheimpflug Topcon SL-45 at Vrije University, Amsterdam. Measurements of this eye were obtained under pupil dilation with tropicamide.

\section{RESULTS}

Figure 3 shows uncorrected raw images from the 
TABLE 2

\section{Nominal and Estimated Radii of Curvature and Interocular Distances From Uncorrected and Corrected Scheimpflug Images for a Human Eye}

\begin{tabular}{lccc}
\hline & Nominal Values $(\mathbf{m m})$ & Before Correction $(\mathbf{m m})$ & After Correction $(\mathbf{m m})$ \\
\hline Anterior corneal radius & 7.72 & 9.43 & 7.86 \\
Corneal thickness & 0.54 & 0.43 & 0.55 \\
Posterior corneal radius & 6.48 & 8.99 & 6.97 \\
Anterior chamber depth & 3.15 & 2.48 & 2.86 \\
Anterior lens radius & 10.54 & 14.68 & 10.37 \\
Lens thickness & 4.04 & 3.83 & 4.06 \\
Posterior lens radius & 5.80 & 8.87 & 5.55 \\
\end{tabular}

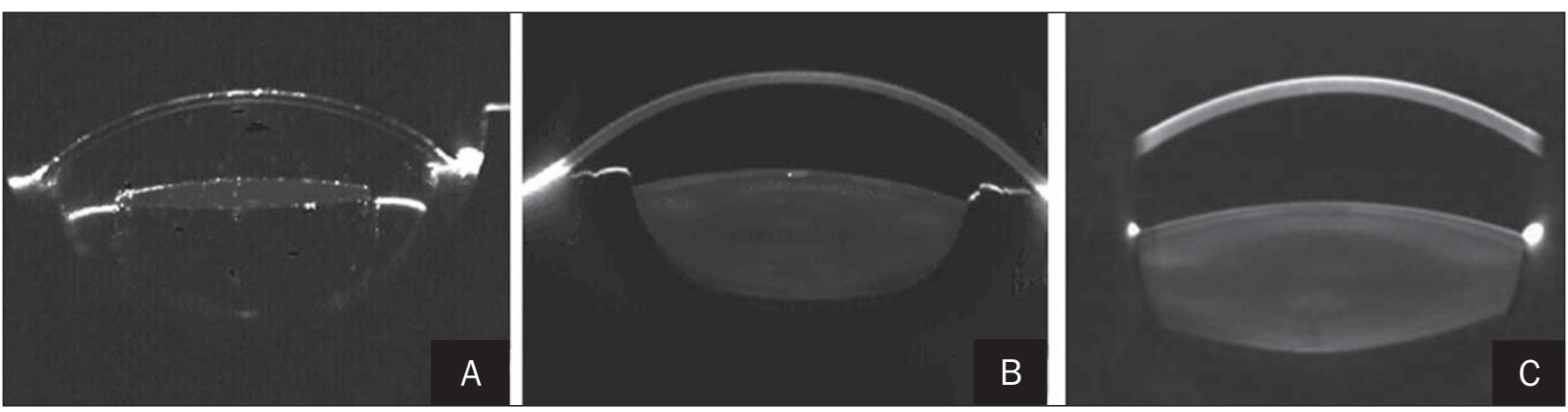

Figure 3. Uncorrected Scheimpflug cross-section images from A) a physical water cell model eye (polymethylmethacrylate cornea and silicone intraocular lens) obtained with the Pentacam system; B) a human young phakic eye obtained with the Pentacam system; and C) the same eye obtained with a refurbished Topcon SL-45 at de Vrije University, Amsterdam.

physical model eye captured with the Pentacam system, and a human phakic eye captured with the Pentacam system and the Topcon SL-45 system. We previously reported the lower visibility of the artificial structures compared to the real cornea and crystalline lens due to a much lower scattering. ${ }^{12}$ Although we could adapt our edge detection algorithms to work on these images, the commercial software of the Pentcam system was unable to properly detect the edges of the artificial lenses.

In Figure 4, circular fits to the detected edges of images $A$ and $B$ have been superimposed in blue dashed lines. Those data have been used in the distortion correction algorithms described previously to reconstruct the corrected surfaces (shown in red dashed lines).

The second and third columns of Table 1 show corneal and lens radii of curvature and lens thickness as obtained directly from uncorrected images and after application of correction algorithms. Table 2 shows the same information for the human eye.

In general, the raw images show much flatter surfaces and thinner structures than in reality. The largest effect of correction occurs for the posterior lens radius of curvature $(109 \%$ and $59 \%$ difference between the uncorrected and corrected image for the artificial and human eye, respectively), although anterior lens, posterior cornea, corneal thickness, and to a lesser extent, lens thickness were also significantly changed with correction.

Figure 5 shows the percentage difference between the nominal values and those obtained from raw and corrected data for both the artificial and human eye. Positive percentage difference values for the radii of curvature are indicative of an overestimation of the radius of curvature (estimated flatter surfaces) and negative values of an underestimation of the radius of curvature (estimated steeper surfaces). Positive percentage difference values for interocular distances are indicative of underestimated values, and negative percentage differences are indicative of overestimated values. In all cases (except for anterior chamber depth), the differences are dramatically reduced after application of the correction-distortion algorithms. Before any correction, the average absolute percentage differences (nominal vs retrieved from raw images) were $37.2 \%$ and $28.6 \%$ for the artificial and human eye, respective- 

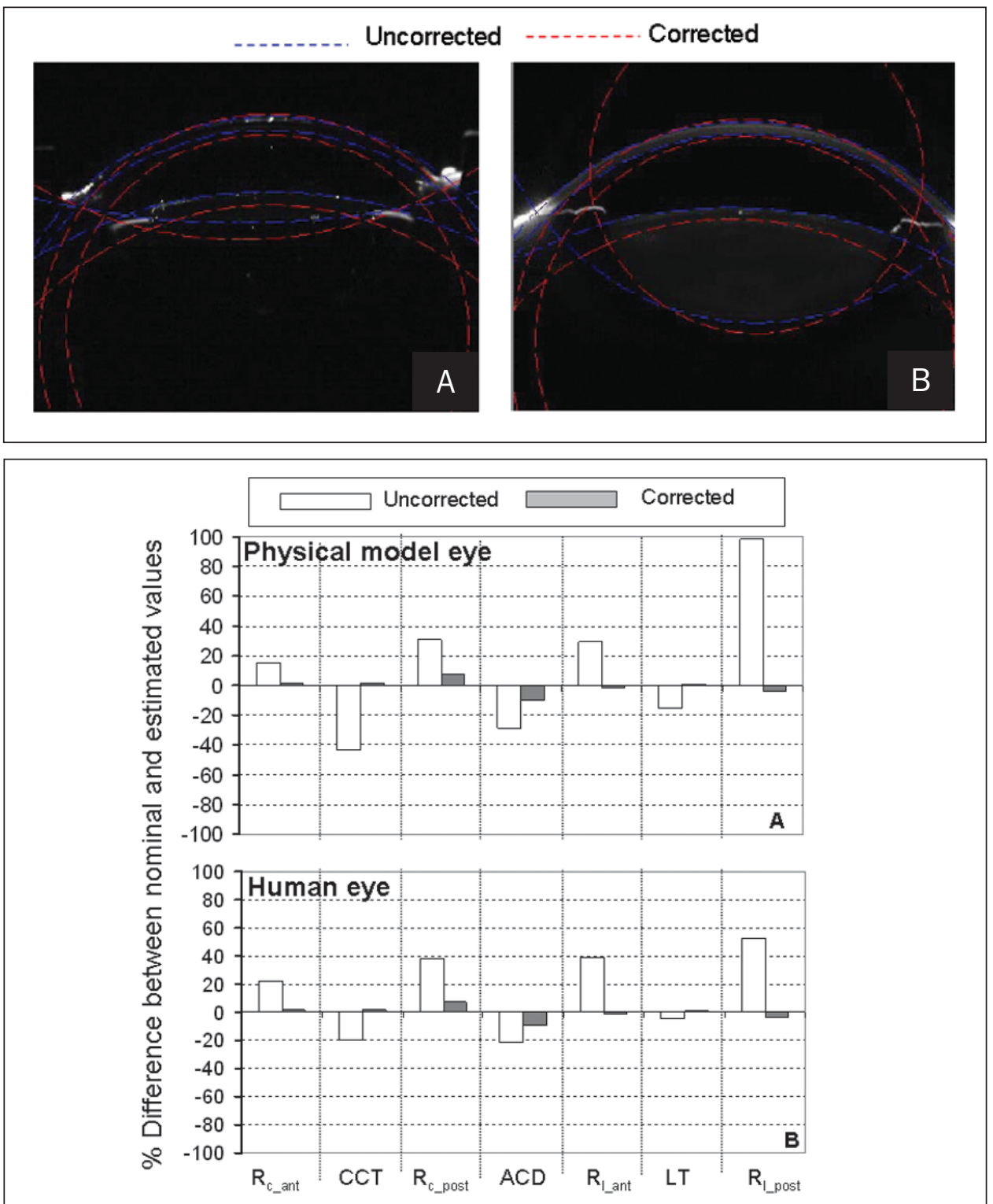

Figure 4. Image processing of data from Figures $3 \mathrm{~A}$ and $3 \mathrm{~B}$. The superimposed lines represent circular fits to the edges of the ocular components before correction (blue lines) and after application of geometrical and optical distortion-correction algorithms (red lines). A) Physical model eye, B) human eye.

Figure 5. Percentage difference between nominal and estimated data of the radius of curvature of the anterior corneal surface $\left(\mathrm{R}_{\mathrm{c} \text { ant }}\right)$, central corneal thickness (CCT), radius of curvature of the posterior corneal surface $\left(R_{c \text { post }}\right)$, anterior chamber depth (ACD), radius of curvature of the anterior lens surface $\left(R_{l_{\text {ant }}}\right)$, lens thickness (LT), and radius of curvature of the posterior lens surface $\left(R_{1 \text { post }}\right)$. White bars represent data from the uncorrected images and gray bars represent data after geometrical and optical distortion correction. Results are for A) the physical model eye and $\mathbf{B}$ ) human eye. Absolute data can be found in Tables 1 and 2 , respectively. ly. When the optical and geometrical distortion correction algorithms are applied, those differences (nominal vs corrected) decrease $5.6 \%$ and $3.8 \%$ (for all values) and $4.0 \%$ and $3.1 \%$ (radii of curvature only) for the artificial and human eye, respectively. We also computed the absolute percentage differences applying a correction of the geometrical distortion only, and found differences (nominal vs corrected) of $16.8 \%$ and $9.2 \%$ (for all values) and $12.8 \%$ and $7.5 \%$ (radii of curvature only) for the artificial and human eye, respectively, indicating that both corrections are essential to provide quantitative information from the images.

\section{DISCUSSION}

We implemented a method for geometrical and optical distortion correction of Pentacam Scheimpflug raw images of the anterior chamber in vivo. This study demonstrates extremely large inaccuracies if corrections are not applied, particularly in the estimates of anterior and posterior lens radii of curvature. This is particularly relevant in current Scheimpflug imagingbased instruments available in the market, as the commercial software typically provides corrections for the anterior and posterior corneal surface but not for the crystalline lens.

We found discrepancies (nomimal-corrected values) of 0.57 and $0.66 \mathrm{~mm}$ in the anterior and posterior lens radii of curvature in the artificial eye, and 0.17 and $0.25 \mathrm{~mm}$ in the human eye. These values were within the reported accuracies of Purkinje imaging ${ }^{14}$ and other Scheimpflug imaging methods. ${ }^{6,11}$ We found lens thickness discrepancies of $0.076 \mathrm{~mm}$ for the IOL and 
$0.02 \mathrm{~mm}$ in the human lens, close to standard ultrasound biometry.

Still, the technique we have described can be subject to several improvements. A deeper knowledge of the optical set-up would have allowed work with real values and not estimations of the camera optics. Our procedure involved retrieval of the object and image distance by means of a least-mean square algorithm. We found that a difference of $50 \mathrm{~mm}(\sim 6 \%)$ in this distance increases the average error from $5.6 \%$ to $10.4 \%$ in the artificial eye and from $3.8 \%$ to $5.3 \%$ in the human eye. It is possible that exact knowledge of the optical layout of the system could further improve the accuracy. Also, in the measurements on the physical eye model, the low scattering in the artificial lenses makes edge detection challenging, and some of the discrepancies may be caused by errors in edge detection, rather than the correction itself. Because the physical model eye was manufactured with spherical surfaces and known refractive index, the estimations are expected to be less affected by simplifications of the reconstruction method. A further sophistication in the algorithm can be the use of aspheric surfaces. Another improvement of the algorithm would involve the use of meridionally variant shapes, rather than conics of revolution. An additional complication in human lens imaging (and correction) is the presence of a gradient refractive index. A typical effective index has been used in the calculations presented here. The impact of a non-homogenous distribution in the optical distortion correction of the posterior lens surface should be further addressed.

We have shown that a commercially available Scheimpflug system can be used to provide quantitative information on the crystalline lens well beyond the current application of the instrument. Correction of optical distortion is critical to obtain reliable phakometry and lens thickness (such as for the posterior corneal elevation map and corneal thickness). Although a Scheimpflug system could not compete in resolution with anterior segment optical coherence tomography (OCT), ${ }^{15}$ which otherwise should also be corrected from optical distortion, ${ }^{16,17}$ the large depth of focus in Scheimpflug images allows full cross-sections of the anterior segment, from the anterior cornea to the posterior lens, in a single snapshot generally not possible with OCT. Applications of corrected Scheimpflug crystalline lens/IOL in vivo imaging include customized eye modeling, ${ }^{18,19}$ studies of quantitative changes of crystalline lens morphology with accommodation, ${ }^{11,20-22}$ aging, ${ }^{6,23}$ or disease, ${ }^{24}$ and assessment of new intraocular implants and surgical approaches for the correction of presbyopia. ${ }^{25,26}$

\section{AUTHOR CONTRIBUTIONS}

Study concept and design (P.R., S.M.); data collection (P.R., S.M.); interpretation and analysis of data (P.R., S.M.); drafting of the manuscript (P.R., S.M.); critical revision of the manuscript (S.M.); statistical expertise (S.M.); obtained funding (S.M.); administrative, technical, or material support (S.M.); supervision (S.M.)

\section{REFERENCES}

1. Ray SF. Applied Photographic Optics. 2nd ed. Oxford, United Kingdom: Focal Press; 1994.

2. Brown N. Slit-image photography and measurement of the eye. Medical \& Biological Illustration. 1973;23:192-203.

3. Sasaki K, Sakamoto Y, Shibata T, Emori Y. The multi-purpose camera: a new anterior eye segment analysis system. Ophthalmic Res. 1990;22(Suppl):3-8.

4. Richards DW, Russell SR, Anderson DR. A method for improved biometry of the anterior chamber with a Scheimpflug technique. Invest Ophthalmol Vis Sci. 1988;29:1826-1835.

5. Kampfer T, Wegener A, Dragomirescu V, Hockwin O. Improved biometry of the anterior eye segment. Ophthalmic Res. 1989;21:239-248.

6. Dubbelman M, van der Heijde GL. The shape of the aging human lens: curvature, equivalent refractive index and the lens paradox. Vision Res. 2001;41:1867-1877.

7. Cook CA, Koretz JF. Methods to obtain quantitative parametric descripions of the optical surfaces of the human crystalline lens from scheimpflug slit-lamp images, I: image processing methods. J Opt Soc Am A Opt Image Sci Vis. 1998;15:1473-1485.

8. Koretz JE, Strenk SA, Strenk LM, Semmlow JL. Scheimpflug and high-resolution magnetic resonance imaging of the anterior segment: a comparative study. J Opt Soc Am A Opt Image Sci Vis. 2004;21:346-354.

9. Dubbelman M, van der Heijde GL, Weeber HA. The thickness of the aging human lens obtained from corrected Scheimpflug images. Optom Vis Sci. 2001;78:411-416.

10. Dubbelman M, Sicam VA, van der Heijde GL. The shape of the anterior and posterior surface of the aging human cornea. Vision Res. 2006;46:993-1001.

11. Rosales P, Dubbelman M, Marcos S, van der Heijde R. Crystalline lens radii of curvature from Purkinje and Scheimpflug imaging. J Vis. 2006;6:1057-1067.

12. de Castro A, Rosales P, Marcos S. Tilt and decentration of intraocular lenses in vivo from Purkinje and Scheimpflug imaging. Validation study. J Cataract Refract Surg. 2007;33:418-429.

13. Norrby S, Artal P, Piers PA, Van Der Mooren M, inventors; Pharmacia Groningen BV, assignee. Methods of obtaining ophthalmic lenses providing the eye with reduced aberrations. US patent 6,609,793. August 26, 2003

14. Rosales P, Marcos S. Phakometry and lens tilt and decentration using a custom-developed Purkinje imaging apparatus: validation and measurements. J Opt Soc Am A Opt Image Sci Vis. 2006;23:509-520.

15. Drexler W, Baumgartner A, Findl O, Hitzenberger CK, Sattmann H, Fercher AF. Submicrometer precision biometry of the anterior segment of the human eye. Invest Ophthalmol Vis Sci. 1997;38:1304-1313.

16. Podoleanu A, Charalambous I, Plesea L, Dogariu A, Rosen R. Correction of distortions in optical coherence tomography imaging of the eye. Phys Med Biol. 2004;49:1277-1294.

17. Westphal V, Rollins A, Radhakrishnan S, Izatt J. Correction of geometric and refractive image distortions in optical coherence tomography applying Fermat's principle. Optics Express. 2002;10:397-404. 
18. Rosales P, Marcos S. Customized computer models of eyes with intraocular lenses. Optics Express. 2007;15:2204-2218.

19. Tabernero J, Piers P, Benito A, Redondo M, Artal P. Predicting the optical performance of eyes implanted with IOLs to correct spherical aberration. Invest Ophthalmol Vis Sci. 2006;47:46514658.

20. Koretz JF, Cook CA, Kaufman PL. Aging of the human lens: changes in lens shape at zero-diopter accommodation. $J \mathrm{Opt}$ Soc Am A Opt Image Sci Vis. 2001;18:265-272.

21. Dubbelman M, van der Heijde GL, Weeber HA. Change in shape of the aging human crystalline lens with accommodation. Vision Res. 2005;45:117-132.

22. Rosales P, Wendt M, Marcos S, Glasser A. Changes in crystalline radii of curvature and lens tilt and decentration during dynamic accommodation in rhesus monkeys. J Vis. 2008;8:1-12.
23. Koretz JF, Cook CA, Kaufman PL. Aging of the human lens: changes in lens shape upon accommodation and with accommodative loss. J Opt Soc Am A Opt Image Sci Vis. 2002;19:144-151.

24. Wiemer NG, Dubbelman M, Kostense PJ, Ringens PJ, Polak BC. The influence of chronic diabetes mellitus on the thickness and the shape of the anterior and posterior surface of the cornea. Cornea. 2007;26:1165-1170.

25. Parel JM, Gelender H, Trefers WF, Norton EW. Phaco-ersatz: cataract surgery designed to preserve accommodation. Graefes Arch Clin Exp Ophthalmol. 1986;224:165-173.

26. Koopmans SA, Terwee T, Barkhof J, Haitjema HJ, Kooijman AC. Polymer refilling of presbyopic human lenses in vitro restores the ability to undergo accommodative changes. Invest Ophthalmol Vis Sci. 2003;44:250-257. 